\title{
Correlation between the Magnetoresistance, IR Magnetoreflectance, and Spin-Dependent Characteristics of Multilayer Magnetic Films
}

\author{
V. G. Kravets \\ Institute for Information Recording, National Academy of Sciences of Ukraine, 2 Shpak Street, Kiev 03113, Ukraine \\ Correspondence should be addressed to V. G. Kravets, vasyl_kravets@yahoo.com
}

Received 27 July 2011; Accepted 25 October 2011

Academic Editor: Manh-Huong Phan

Copyright (C) 2012 V. G. Kravets. This is an open access article distributed under the Creative Commons Attribution License, which permits unrestricted use, distribution, and reproduction in any medium, provided the original work is properly cited.

\begin{abstract}
We present the experimental results on magnetorefractive effect (MRE) in ferromagnetic metal-metal and metal-insulator multilayer films of different composition and different type of magnetoresistive effects. The shape and magnitude of the MRE dependences are found to be very sensitive to the spin-dependent scattering parameters and the effective polarization of the electron density of state around the Fermi level. A study of an MRE in multilayered films is shown to be sufficient for direct extracting of the spin-dependent relaxation times of electron (for GMR-like samples) and energy dependence of the tunnel spin-polarization density of states near the Fermi level for layered TMR films. It is proposed to use the magnetorefractive effect as a noncontact probe of magnetoresistive effects in thin magnetic films through investigations of the field-dependent reflection behaviors of multilayered films in the IR region.
\end{abstract}

\section{Introduction}

The discovery $[1,2]$ of the giant magnetoresistance (GMR) effect has led to many technological applications, especially in data storage devices such as GMR read heads, magnetoresistive random access memory, and in magnetic sensors $[3,4]$. The GMR effect reflects changes in resistivity as result of spin-dependent scattering of two spin channels across the interfaces between the magnetic and nonmagnetic metal layers (i.e., GMR effect) or magnetic and insulating layers (i.e., tunneling magnetoresistance) [5]. The key property of these materials is the reduction of their electrical resistivity in magnetic field. Tunneling magnetoresistance effect (TMR) draws much attention for its fascinating transport properties and industrial applications $[4,5]$.

The most common method of measuring magnetoresistance (MR) involves passing an electrical current through the sample via a four-point probe. On the other hand, Jacquet and Valet [6] proposed and demonstrated an alternative noncontact method for the measurement of magnetotransport effects using electromagnetic radiation of infrared (IR) spectral region. It relies on the fact that at IR wavelengths the optical properties in metals depend mainly on electron transition within the conduction band (intraband transitions). The method is based on the magnetorefractive effect (MRE). The MRE is the variation of the complex refractive index (dielectric function) of a material due to a change in its conductivity at IR frequencies when a magnetic field is applied. MRE opens a new stage in magnetooptics (MO) because it is not connected with spin-orbit interaction. MRE is an even MO effect, namely, it is linear with magnetization squared, like GMR. Moreover, the MRE is sufficiently larger in the IR region than the corresponding MO values [6-8]. Infrared transmission or reflection spectroscopy can provide a direct tool for probing the spin-dependent conductivity in GMR and TMR samples [6-11].

The MRE of ferromagnetic/nonmagnetic metal multilayer and granular films has been a field of intensive studies over the last decade. This interest in MRE was motivated by three aspects: (i) its importance as an experimental noncontact (in situ) magnetoresistance tool, (ii) its being a means of measuring the spin-dependent characteristics of ferromagnetic (FM) materials, and (iii) its application in magnetooptical crystal. The main advantages of the MRE 
over other techniques are following: (i) the surface sensitivity with typical information depth of $\sim 10-30 \mathrm{~nm}$; (ii) a time resolution can be down to the subpicosecond regime $[6,8]$; (iii) a reasonable spatial resolution tends to $\sim 1-10 \mu \mathrm{m}[6$, 11]; (iv) using robust and inexpensive experimental setups.

The full quantitative MRE information is generally not linked by simple analytic formulas to the material properties, which are the dielectric function of all involved layers and their GMR or TMR values. Moreover, the literature is almost exclusively limited to the MRE in multilayer and granular films with GMR effect. The magnetotransport characterization of metal-dielectric multilayers (TMRs) is more difficult than continuous metallic multilayer (GMR), and hence there is a great tendency to develop a noncontact method for studying their electrical behaviors. The spin-dependent characteristics extracted from modeling of the MRE are also important in fundamental research. From a microscopic point of view the MRE in layered TMR films is due to the interplay of the exchange interaction leading to a splitting of the bands and the spin-dependent density of states around the Fermi level.

In this work we have measured the MRE of $\mathrm{CoFe} / \mathrm{Cu}$ multilayer films with GMR effect and of $\mathrm{CoFe} / \mathrm{Al}_{2} \mathrm{O}_{3}$ films with TMR effect. Measurements are made in reflection mode which is the most likely mode for practical remote sensing of magnetoresistance effects. The good agreement at middle IR wavelengths between electrical magnetotransport measurements and noncontact magnetorefractive effect demonstrates the possibilities of using IR reflection spectroscopy for the characterization of giant magnetoresistive systems. We have chosen the multilayer structures with low values of magnetoresistance for checking the sensitivity of magnetorefractive effect. It was shown that the spin-dependent scattering times $\tau^{\dagger}$ and $\tau^{\downarrow}$ (or mean free path) of majority and minority electrons can be directly extracted from the MRE dependences in the IR region. We also demonstrate how a low-energy spectrum of the tunnel spin-dependent density of states around of the Fermi level for layered TMR films can be reconstructed from its magnetoreflective spectrum. This can be done by modeling the MRE dependences as an effective polarization, $P_{\text {eff }}(E)$, and extracting the optical conductivities of the different layers from the reflection spectra. Our results demonstrate direct schedule for obtaining the energy dependence of the spin-polarization density of states near the Fermi level.

\section{Experimental Techniques}

The choice of the $\mathrm{CoFe} / \mathrm{Cu}\left(\mathrm{Al}_{2} \mathrm{O}_{3}\right)$-based film structures was motivated by the fact that spin polarization of the $\mathrm{CoFe}$ alloy exceeds those in $\mathrm{Co}$ and $\mathrm{Fe}$, while nonmagnetic $\mathrm{Cu}$ demonstrates excellent electrical conductivity and insulating $\mathrm{Al}_{2} \mathrm{O}_{3}$ provides a low tunneling barrier and high values of the TMR. The multilayer films were prepared by dc magnetron sputtering at $\sim 10^{-4}$ Torr Ar gas pressure. The films were deposited on glass and Si substrates using two targets of CoFe and $\mathrm{Cu}$ or $\mathrm{Al}_{2} \mathrm{O}_{3}$. For the ferromagnetic layers we used $\mathrm{Co}_{75} \mathrm{Fe}_{25}$ target which is a soft magnetic alloy. The room temperature GMR was measured using standard four-point probe technique in magnetic fields of up to $8 \mathrm{kOe}$ with both current and applied magnetic field $H$ in the films plane. For TMR measurements a $50 \mathrm{~nm}$ thick CoFe layer was deposited onto glass $(\mathrm{Si})$ substrates in the form of stripe $10 \times$ $20 \mathrm{~mm}^{2}$. Then the $\left[\mathrm{CoFe}(1.6 \mathrm{~nm}) / \mathrm{Al}_{2} \mathrm{O}_{3}(3 \mathrm{~nm})\right]_{10}$ multilayer structures were sputtered onto the center of the CoFe $(50 \mathrm{~nm})$ film in form of stripe $10 \times 10 \mathrm{~mm}^{2}$. On top of devices second electrode CoFe $(50 \mathrm{~nm})$ was formed in the rectangular shape $10 \times 20 \mathrm{~mm}^{2}$ perpendicular to the long axis of the first thick CoFe layer. In process of TMR measurements the magnetic field was oriented perpendicular to the current direction and parallel to the film plane.

A Bruker IFS 113 Fourier transform spectrometer was employed to study reflectance spectra of multilayer structures at $300 \mathrm{~K}$ in the mid-IR range from 2.0 to $25 \mu \mathrm{m}$. The IR radiation was focused onto the sample placed in the electromagnet gap. The magnetoreflectance was determined by analogy with the magnetoresistive effect: $\Delta \rho / \rho=(\rho(H)-$ $\rho(H=0)) / \rho(H=0)$, and $\Delta R / R=(R(H)-R(H=0)) /$ $R(H=0)$. These functions describe the changes in the electrical resistivity $\Delta \rho / \rho$ and light reflectivity $\Delta R / R$ of a material in an external magnetic field. The magnetic field was oriented perpendicular to the IR propagation direction and parallel to the film plane. The magnetic field strength was varied from 0 to $4.0 \mathrm{kOe}$. The angle of incidence of light on the sample was closed to $\sim 45^{\circ}$. The incoming light was polarized in plane of incidence ( $p$-polarization) using a KRS-5 grid polarizer. The IR reflection spectra were taken for all samples at least 100 scans in the spectral range $2.0-25 \mu \mathrm{m}$ and with resolution of $4 \mathrm{~cm}^{-1}$.

\section{Experimental Results and Their Discussions}

The MRE spectra of the $[\operatorname{CoFe}(2 \mathrm{~nm}) / \mathrm{Cu}(1.2 \mathrm{~nm})]_{10}$ multilayers are presented in Figure 1(a). These spectra have a broad positive peak at short wavelengths $(2-6 \mu \mathrm{m})$ followed by a crossover into a reduced reflection region resulting in negative MRE at longer wavelengths. $\Delta R / R$ spectra of CoFe/ $\mathrm{Cu}$ multilayer films reveal deep minima at $\lambda \sim 20-21 \mu \mathrm{m}$ (Figure 1(a)). As the magnetic field increases from 1.2 to $3.75 \mathrm{kOe}, \Delta R / R$ grows in absolute magnitude nonlinearly from $\sim 0.3$ to $\sim 1.1 \%$. The decrease of the reflectivity in an applied magnetic field is consistent with the increase in electrical conductivity resulting from the magnetically induced modification of the scattering of the Drude-like free electrons. The lowest value of the MRE spectrum located at a wavelength $\lambda \sim 20.7 \mu \mathrm{m}$, which is far away from the short wavelength region where interband transitions are important.

Figure 1(b) shows the dependence of MRE for $\left[\mathrm{CoFe}(1.6 \mathrm{~nm}) / \mathrm{Al}_{2} \mathrm{O}_{3}(3 \mathrm{~nm})\right]_{10}$ multilayer films. The MRE goes down with increasing wavelength of the incident IR probe light and reaches the maximal negative value at wavelengths $\lambda \sim 13-14 \mu \mathrm{m}$. It can be seen that absolute value of the MRE tends to value of $\sim 0.5 \%$ in the magnetic field of $H=3.75 \mathrm{kOe}$. Note that the MRE demonstrates a nonlinear dependence on the magnetic field $H$ and the function $\Delta R / R$ becomes positive (changes its sign) in the low wavelength region $2.0-5 \mu \mathrm{m}$ due to contribution of the interband 


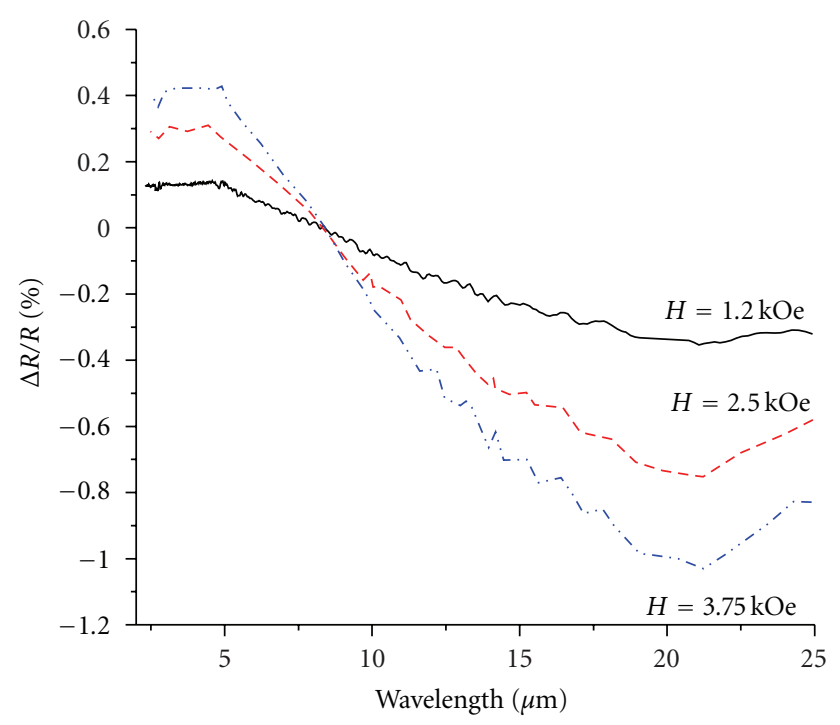

(a)

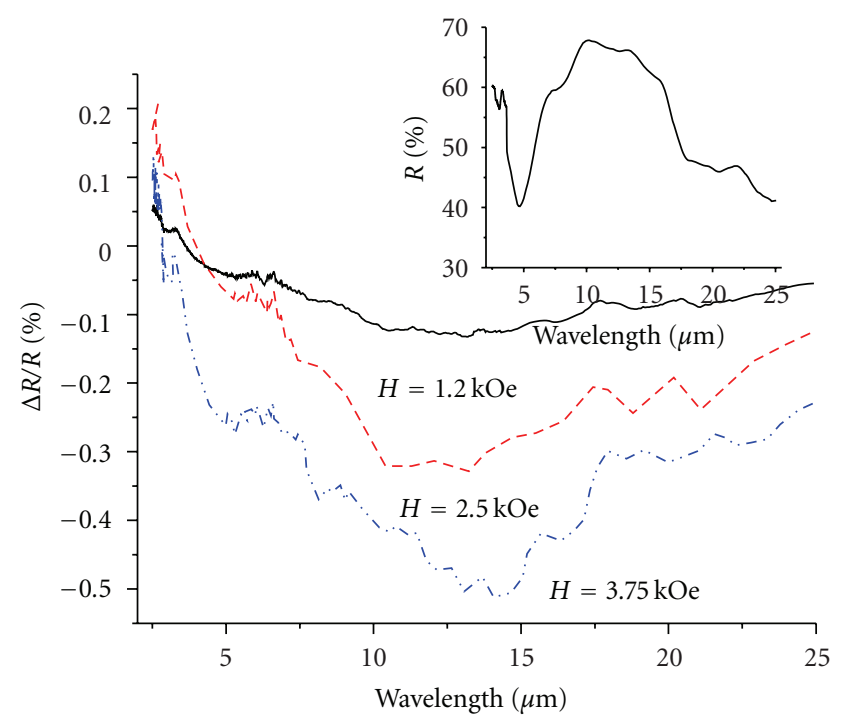

(b)

FIgure 1: The experimental MRE spectra for [CoFe $(2 \mathrm{~nm}) /$ $\mathrm{Cu}(1.2 \mathrm{~nm})]_{10}$ (a) and $\left[\mathrm{CoFe}(1.6 \mathrm{~nm}) / \mathrm{Al}_{2} \mathrm{O}_{3}(3 \mathrm{~nm})\right]_{10}$ (b) multilayer films, $H=1.2,2.5$, and $3.75 \mathrm{kOe}$. Inset of (b): the IR reflection spectra of the $\mathrm{CoFe} / \mathrm{Al}_{2} \mathrm{O}_{3}$ multilayer films.

electron transitions. Thus, the competition between the intraband and interband contributions leads to a change of sign of MRE as a function of wavelength. This experimental fact contradicts to the predictions of the Drude model [610 ], where the intraband contribution into the conductivity is considered only. Due to such contribution the MRE curve always keeps the same sign. It means that the main mechanism of electron transport in the metal-metal and metalinsulating multilayer structures is the s-d exchange scattering [5]. The shape of the MRE curves as a function of wavelength is therefore sensitive to the s- and d-dependent electron scattering parameters, which determine the GMR and TMR effects.
The IR reflection spectra of the $\mathrm{CoFe} / \mathrm{Al}_{2} \mathrm{O}_{3}$ multilayer films (inset of Figure 1(b)) display the complex structure associated with the phonon vibrations in oxide layers. For the spectral region $5-20 \mu \mathrm{m}$ the reflectivity $R(\lambda)$ of multilayers becomes similar to the spectrum of the pure $\mathrm{Al}_{2} \mathrm{O}_{3}$. Reststrahlen band of high reflectivity between $\sim 5$ and $20 \mu \mathrm{m}$ is visible. $R(\lambda)$ spectra show pronounced features at $\lambda \sim 5.0$ 7.5 and $17-18 \mu \mathrm{m}$ which can be assigned to the longitudinal optical (LO) and transverse optical (TO) vibration modes of aluminium oxide which is in agreement with previous studies [11]. It should be pointed out that the value of $\lambda_{\mathrm{LO}}$, where the reflectivity tends to its minimum, is shifted to lower wavelengths with respect to $\mathrm{Al}_{2} \mathrm{O}_{3}$, and the reflectivity between LO and TO modes does not reaches the value $R \approx$ $100 \%$.

Figure 2 shows the high correlation between the electrical measured GMR (TMR) and the optically measured MRE for $\mathrm{CoFe} / \mathrm{Cu}$ and $\mathrm{CoFe} / \mathrm{Al}_{2} \mathrm{O}_{3}$ multilayer films. The differences in the nonmagnetic layer $\mathrm{Cu}$ or $\mathrm{Al}_{2} \mathrm{O}_{3}$ lead to different values of the magnetoresistive effect (Figure 2(a)). The maximum value of magnetoresistance of about $\sim 12.5 \%$ and $\sim 2.5 \%$ in the magnetic field $H=8.2 \mathrm{kOe}$ is observed for $\mathrm{CoFe} / \mathrm{Cu}$ and $\mathrm{CoFe} / \mathrm{Al}_{2} \mathrm{O}_{3}$ multilayer films, respectively. The measurements of the MRE profiles were carried out at fixed wavelength when the magnetoreflectance reaches a maximum. The experiment was performed by direct recording of the changes in the reflected intensity as a function of applied magnetic field at $\lambda \sim 20.7 \mu \mathrm{m}$ and $\lambda \sim 13.5 \mu \mathrm{m}$ for $\mathrm{CoFe} / \mathrm{Cu}$ and $\mathrm{CoFe} / \mathrm{Al}_{2} \mathrm{O}_{3}$ multilayer films, respectively (Figure 2(b)). It has been previously shown $[9,11]$ that the correlation between the GMR and MRE should be most evident at extreme wavelengths where the MRE reproduces the maximum values. These dependences (Figures 2(a) and 2(b)) clearly show the possibility to perform noncontact magnetotransport measurements on the samples with giant and tunneling magnetoresistance by measuring the variation of their reflected intensity in magnetic field at fixed wavelength, $\lambda$. Note that the absolute magnitude of MRE in $\mathrm{CoFe} / \mathrm{Cu}$ multilayer films is higher than that in $\mathrm{CoFe} / \mathrm{Al}_{2} \mathrm{O}_{3}$ films and this trend is consistent with GMR (TMR) dependences.

\section{Theoretical Treatments}

4.1. MRE Effect in Metallic Magnetoresistive Samples with $G M R$. To interpret our results we have performed simulations of the MRE for two different systems and their dependence on the scattering and carries characteristics of the different type of magnetoresistance. The calculations of the reflection, transmission, and absorption spectra are performed using a $2 \times 2$ characteristic transfer matrix for $N$ number of parallel, homogeneous, isotropic layers [13]. This formalism takes into account the multiple reflections on the interfaces of the multilayer film and could also be used to calculate the MRE in different multilayer structures. The optical response of every layer describes by a complex effective index of refraction. We have simulated the MRE of $[\mathrm{CoFe}(2 \mathrm{~nm}) / \mathrm{Cu}(1.2 \mathrm{~nm})]_{10}$ multilayers using the frequency- and spin-dependent conductivity for FM film. Conductivity $\sigma(\omega)$ of the FM layer is a sum of the Drude 


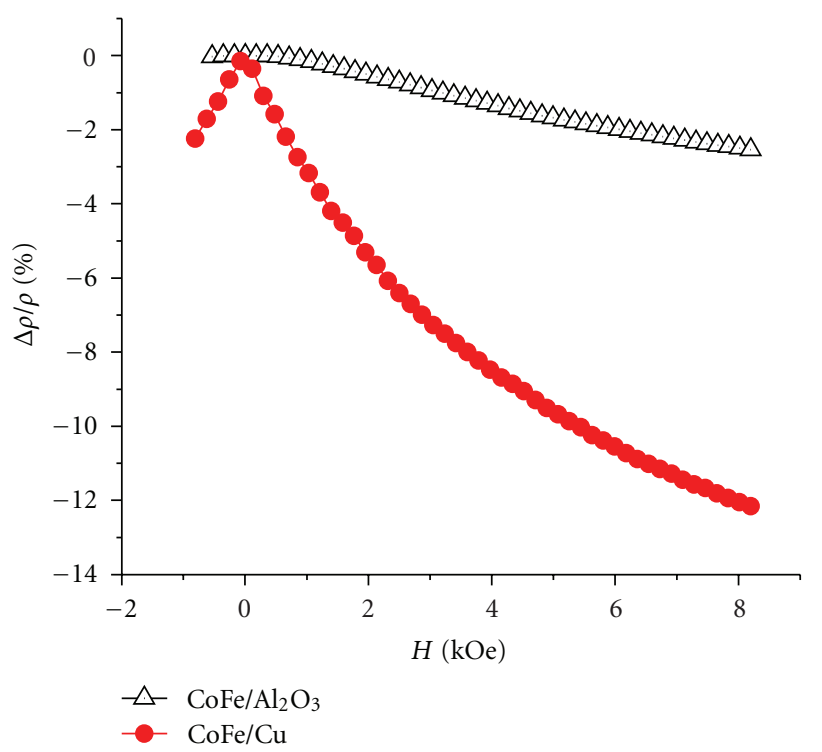

(a)

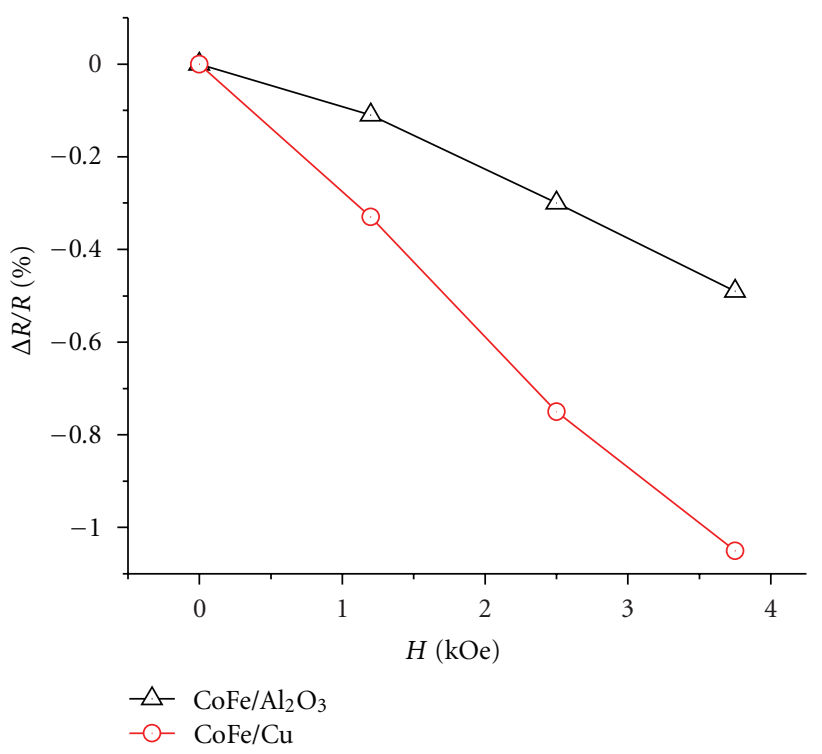

(b)

Figure 2: (a) The experimental $\Delta \rho / \rho$ data of $[\mathrm{CoFe}(2 \mathrm{~nm}) /$ $\mathrm{Cu}(1.2 \mathrm{~nm})]_{10}$ and $\left[\mathrm{CoFe}(1.6 \mathrm{~nm}) / \mathrm{Al}_{2} \mathrm{O}_{3}(3 \mathrm{~nm})\right]_{10}$ multilayer films; (b) measured MRE versus magnetic field at fixed wavelength $(\lambda=$ $20.7 \mu \mathrm{m})$ for $[\mathrm{CoFe}(2 \mathrm{~nm}) / \mathrm{Cu}(1.2 \mathrm{~nm})]_{10}$ and $(\lambda=13.5 \mu \mathrm{m})$ for $\left[\mathrm{CoFe}(1.6 \mathrm{~nm}) / \mathrm{Al}_{2} \mathrm{O}_{3}(3 \mathrm{~nm})\right]_{10}$ multilayer films.

contributions from majority- and minority-spin electrons $[7,8,14]$ :

$$
\sigma(\omega)=\sigma^{\dagger}(\omega)+\sigma^{\downarrow}(\omega)=\frac{\sigma^{\dagger}(\omega=0)}{1+i \omega \tau^{\dagger}}+\frac{\sigma^{\downarrow}(\omega=0)}{1+i \omega \tau^{\downarrow}}
$$

in which $\sigma^{\uparrow(\downarrow)}(\omega=0)$ and $\tau^{\uparrow(\downarrow)}$ are the spin-dependent $\mathrm{dc}$ conductivity and relaxation times, respectively. This conductivity is given by $\sigma^{\dagger(\downarrow)}(\omega=0)=n^{\dagger(\downarrow)} e^{2} \tau^{\dagger(\downarrow)} / m$, where $n^{\dagger(t)}$ are spin-dependent conduction electron density and $m$ is the mass of an electron. In ferromagnetic alloy CoFe electronic bands are exchange split, which implies different
Fermi wavevectors for the spin-up and spin-down electrons, $k_{F}^{\dagger(\downarrow)}$. The values $k_{F}^{\dagger} \sim 7.5 \mathrm{~nm}^{-1}$ and $k_{F}^{\downarrow} \sim 4.5 \mathrm{~nm}^{-1}$ were determined assuming a free electron gas $[15,16]$, and the magnitude of $n^{\dagger(\downarrow)}$ can be estimated from the relation $n^{\dagger(\downarrow)}=$ $\left(k_{F}^{\dagger(\downarrow)}\right)^{3} / 3 \pi^{2}$. We have introduced the spin-asymmetry parameter $\alpha_{0}$, which is defined as the ratio between the spinup and spin-down relaxation times for zero magnetic field, $\alpha_{0}=\tau_{0}^{\dagger} / \tau_{0}^{\downarrow}$. These average spin-dependent scattering times for spin-up and spin-down connect to the spin-independent scattering time, $\tau_{f}$, in the ferromagnetic CoFe: $\tau_{f}^{-1}=$ $\left(\left(\tau_{0}^{\dagger}\right)^{-1}+\left(\tau_{0}^{\downarrow}\right)^{-1}\right) / 2$. For fitting experimental results the following parameters were chosen according to literature data $[8,12,14]: \tau_{f}=1.25 \times 10^{-14} \mathrm{~s}$ closed to the relaxation time of pure Co, $\alpha_{0}=3$ the spin-asymmetry parameter, $\tau_{n}=$ $\tau_{\mathrm{Cu}}=2.5 \times 10^{-14} \mathrm{~s}$ the spin-independent scattering time in the nonmagnetic layer, $\mathrm{Cu}$. In the external magnetic field the spin-dependent relaxation time changes as a $\tau^{\dagger(\downarrow)}=\tau_{0}^{\dagger(\downarrow)}(1 \pm$ $\alpha)$. The parameter $\alpha$ is connected to the GMR: $\Delta \rho / \rho=\alpha_{0} \alpha-$ $\alpha /\left(\alpha_{0}+1\right)$. This expression for $\Delta \rho / \rho$ results from (1) at $\omega=0$, including the spin-asymmetry parameter $\alpha_{0}$. Thus we have all parameters to calculate the spin-dependent conductivity in the middle IR region for ferromagnetic materials. In the next step, the Drude's model was employed to find the complex refractive index for $\mathrm{CoFe}$ and $\mathrm{Cu}$ layers:

$$
\begin{gathered}
n_{f}-i k_{f}=\sqrt{\varepsilon_{f r}-\frac{i \sigma(\omega)}{\varepsilon_{0} \omega}}, \\
n_{n}-i k_{n}=\sqrt{\varepsilon_{n r}-\frac{i \omega_{p}^{2} \tau_{n}}{\omega\left(1-i \omega \tau_{n}\right)}} .
\end{gathered}
$$

Here, $\omega_{p}=1.0 \times 10^{16} \mathrm{~s}^{-1}$ is the "quasifree" plasma frequency of $\mathrm{Cu}[12], \varepsilon_{0}=8.85 \times 10^{-12} \mathrm{As} / \mathrm{Vm}$ is the permittivity of free space, $\varepsilon_{f r}$, and $\varepsilon_{n r}$ is the relative dielectric constant of the CoFe and $\mathrm{Cu}$ layer, respectively, which is contributed by bound electrons. For the highly conducting films used in our model we can set values $\varepsilon_{f r}, \varepsilon_{n r}$ equal to 1 ; they do not influence on the complex optical constants of ferromagnetic $\mathrm{CoFe}, n_{f}-i k_{f}$ and nonmagnetic $\mathrm{Cu}, n_{n}-i k_{n}$ films.

Figure 3(a) shows the theoretical MRE spectra for $\mathrm{CoFe} / \mathrm{Cu}$ multilayer films. It is shown that the wavelengths dependence of $\Delta R / R$ can be explained quite well, and our fit is satisfactory for spectral region 2-25 $\mu \mathrm{m}$. In order to exactly reproduce the shape of MRE we modified the effective Drude parameters. According to [17] the spin-dependent relaxation time $\tau_{0}^{\dagger(\downarrow)}$ is multiplied by function of $\left[\left(\hbar \omega-E_{F}^{\dagger(\downarrow)}\right) / E_{F}^{\dagger(\downarrow)}\right]^{2}$ which includes the contributions from interband electrons transitions into MRE effect. Due to such dependence the MRE changes the sign from positive to negative values at short wavelengths.

The shape of the MRE curves as a function of wavelength is more sensitive to the spin-dependent scattering parameters that determine the GMR. The most important parameters in determining the MRE response are $\omega \tau^{\dagger(\downarrow)}$, the product of the angular frequency of the IR radiation and relaxation times appropriate to spin-up and spin-down. It is instructive to consider typical values of the critical parameter $\omega \tau^{\dagger(\downarrow)}$ at 


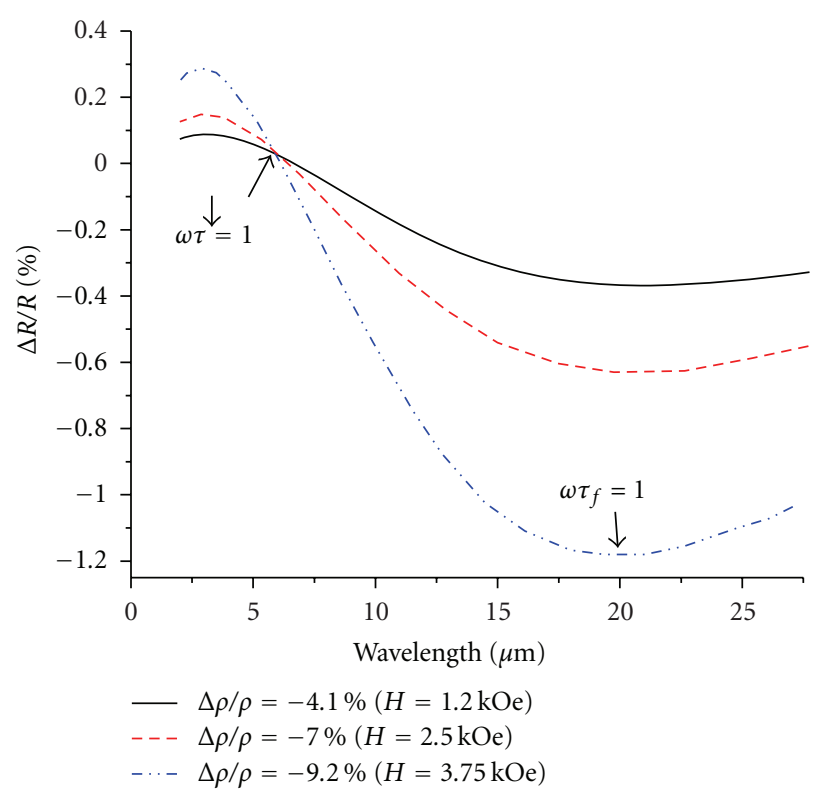

(a)

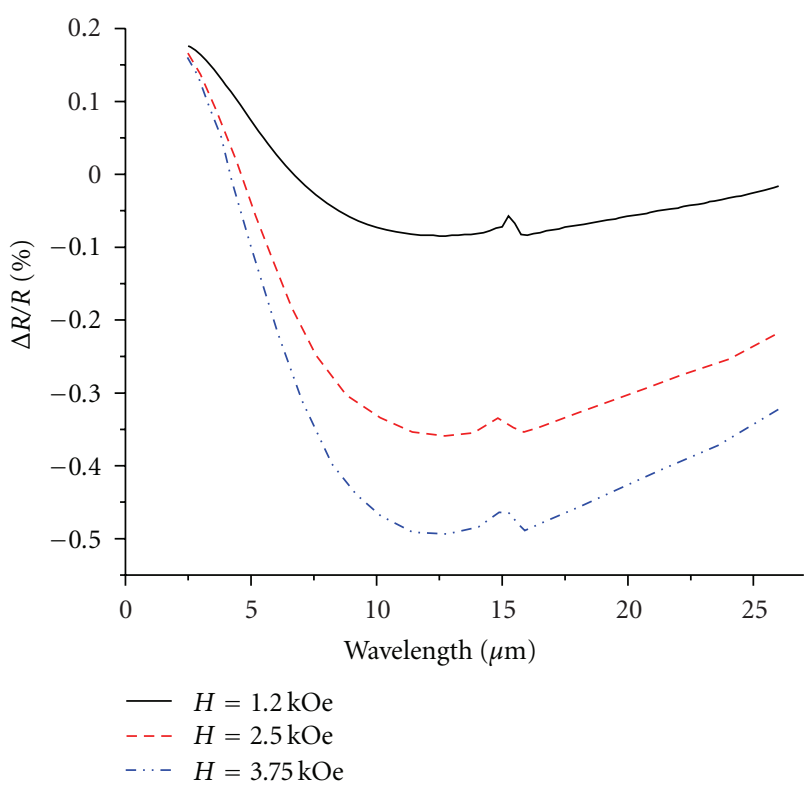

(b)

Figure 3: The theoretical MRE spectra for [CoFe $(2 \mathrm{~nm}) /$ $\mathrm{Cu}(1.2 \mathrm{~nm})]_{10}$ (a) and $\left[\mathrm{CoFe}(1.6 \mathrm{~nm}) / \mathrm{Al}_{2} \mathrm{O}_{3}(3 \mathrm{~nm})\right]_{10}$ (b) multilayer films, $H=1.2,2.5$, and $3.75 \mathrm{kOe}$.

certain points in the MRE curves. From theoretical point of view $[10,18]$ the MRE curves are predicted to cross zero values at the frequencies: $\omega_{1} \tau^{\dagger} \approx 1$ and $\omega_{2} \tau^{\downarrow} \approx 1$. From dependences represented in Figure $1($ a) we can estimate the value of $\tau^{\downarrow} \approx 4.4 \times 10^{-15} \mathrm{~s}$. As the wavelength is increased the MRE reaches the maximal absolute value where $\omega \tau_{f} \approx$ 1. The extracted value of $\tau_{f} \approx 1.12 \times 10^{-14} \mathrm{~s}$ is consistent with literature data $\left(\tau_{f} \approx 1.25 \times 10^{-14} \mathrm{~s}[12]\right)$. The measured spectral region is restricted by $25 \mu \mathrm{m}$, and the MRE curves do not cross again the zero value where
TABLE 1: Comparison of the theoretical and extracted from MRE spectra spin-dependent parameters for the $\mathrm{CoFe} / \mathrm{Cu}$ multilayer films.

\begin{tabular}{lcc}
\hline Parameters & $\begin{array}{c}\text { Theoretical data } \\
{[8,12]}\end{array}$ & $\begin{array}{c}\text { Experimental data } \\
(\mathrm{MRE})\end{array}$ \\
\hline $\begin{array}{l}\text { Average scattering } \\
\text { time: } \tau_{f}\end{array}$ & $1.25 \times 10^{-14} \mathrm{~s}$ & $1.12 \times 10^{-14} \mathrm{~s}$ \\
$\begin{array}{l}\text { Spin-dependent } \\
\text { scattering times: } \\
\text { spin-up } \tau^{\dagger}\end{array}$ & $1.6 \times 10^{-14} \mathrm{~s}$ & $1.1 \times 10^{-14} \mathrm{~s}$ \\
spin-down $\tau^{\downarrow}$ & $4.6 \times 10^{-15} \mathrm{~s}$ & $4.4 \times 10^{-15} \mathrm{~s}$ \\
\hline
\end{tabular}

$\omega_{1} \tau^{\dagger} \approx 1$. This result shows immediately the effect of the two separate spin directions on the total MRE spectra. The relationship between the relaxation time and GMR depends on the specific scattering mechanism; for example, a high GMR may be the result of a very long $\tau^{\uparrow}$ or a very short $\tau \downarrow$. The comparison between theoretical and experimental spin-dependent scattering parameters as extracted from the experimental MRE are presented in Table 1. Thus, we have shown that the known dependence of MRE enables a direct extraction of spin-dependent relaxation times $\tau^{\dagger(\downarrow)}$ (or mean free path) without fitting parameters. Such extraction of $\tau_{0}{ }^{\dagger(\downarrow)}$ is possible due to crossing the MRE curves of zero values.

4.2. MRE Effect in $\mathrm{Al}_{2} \mathrm{O}_{3}$-Based Magnetic Tunnel Junctions. We now turn to the investigation and discussion of the $\mathrm{MRE}$ in $\left[\mathrm{CoFe}(1.6 \mathrm{~nm}) / \mathrm{Al}_{2} \mathrm{O}_{3}(3 \mathrm{~nm})\right]_{10}$ multilayer films with TMR effect. TMR originates from the difference in the density of states (DOS) at the $E_{F}$ between spin-up $N_{\uparrow}\left(E_{F}\right)$ and spin-down $N_{\downarrow}\left(E_{F}\right)$ electrons. In order to fit the TMR data (Figure 2(a)) we have used the known Julliere law for multilayer films [19]. According to this model of spin polarized tunneling $[19,20]$, TMR is given by

$$
\frac{\Delta \rho}{\rho}=\frac{\rho(H)-\rho(0)}{\rho(0)}=\frac{2 P_{1} P_{2}}{1+P_{1} P_{2}},
$$

where $\rho(0)$ and $\rho(H)$ are the junction resistance for the antiparallel and parallel magnetisation configurations of the two ferromagnetic layers, respectively. $P_{1}$ and $P_{2}$ are the spin polarization of the two FM materials (for our structures, $\left.P_{1}=P_{2}\right): P=\left(N_{\uparrow}-N_{\downarrow}\right) /\left(N_{\uparrow}+N_{\downarrow}\right)$. From modelling the TMR data (3) we obtained the effective tunnel spin-polarization $P_{\text {eff }}$ equal to $\sim 11 \%$. This value is lower than the spin-polarization of $\mathrm{Fe}(P \sim 44 \%)$ and $\mathrm{Co}(P \sim 35 \%)$, and $\mathrm{Co}_{50} \mathrm{Fe}_{50}(P \sim 55 \%)$ films, respectively [20]. The lower values of $P_{\text {eff }}$ are probably the result of following factors. (1) The tunnelling spin polarization is not only determined by the properties of the FM layers but also depends on the electronic structure of the insulator and ferromagnetic/insulator interfaces. (2) A number of different processes may contribute to the variation of TMR, such as the spin-dependent electronic structure of the FM layers, inelastic scattering by defect/impurity states in the barrier, and electron-phonon, electron-magnon, and electron-electron interactions. The decrease in TMR is also caused by 
oxidization of FM layers and roughness of surface at interfaces.

Our investigation of the MRE of $\mathrm{CoFe} / \mathrm{Al}_{2} \mathrm{O}_{3}$ multilayer has shown that magnetoreflectance $(\Delta R / R)$ is more sensitive to the abovementioned factors than magnetoresistance $(\Delta \rho / \rho)$. TMR as a function of $P_{1,2}$ is independent from energy (the values $P_{1,2}$ are taken at fixed energy, $E_{F}$ ). In measured MRE dependences for layered TMR films the functions $P_{1,2}$ stay energy dependent. To model the MRE in CoFe/ $\mathrm{Al}_{2} \mathrm{O}_{3}$ multilayer films, it can be logical to consider the spindependent conductivity of two FM layers (1). The tunnel conductivity increases when the magnetizations of the two FM layers are parallel than when they are antiparallel. Analyzing the results in the same way as for TMR the difference in conductivity can be defined as

$$
\frac{\Delta \sigma(\omega)}{\sigma(\omega)}=\frac{\sigma(\omega, H)-\sigma(\omega, H=0)}{\sigma(\omega, H)}=-\frac{2 P_{1}(\omega) P_{2}(\omega)}{1+P_{1}(\omega) P_{2}(\omega)},
$$

where the polarizations $P_{1}(\omega)=P_{2}(\omega)=P(\omega)$ are connected to $\Delta \rho / \rho$ at $\omega=0$ (3). Since spin-flip scattering is neglected, the total conductivity is comprised of two independent electron-tunneling transitions associated with two spin populations.

According to the Hagen-Rubens relation [7, 14], the GMR (TMR) and MRE effects are connected to each other and the reflectivity is a function of the conductivity at low energies, $\sigma(\omega), R=1-2\left[2 \varepsilon_{0} \omega / \sigma(\omega)\right]^{1 / 2}$, where $\varepsilon_{0}$ is the permittivity of free space. The change in reflectivity $\Delta R / R$ can be obtained as a first derivation of $R(\lambda)$ :

$$
\frac{\Delta R}{R}=\frac{\left[2 \varepsilon_{0} \omega / \sigma(\omega)\right]^{1 / 2}}{1-\left[2 \varepsilon_{0} \omega / \sigma(\omega)\right]^{1 / 2}} \times \frac{\Delta \sigma(\omega)}{2 \sigma(\omega)}=-\frac{1-R}{R} \times \frac{\Delta \sigma(\omega)}{2 \sigma(\omega)} .
$$

It is evident from (5) that $\Delta R / R$ depends on magneto-conductivity $(\Delta \sigma(\omega) / \sigma(\omega))$. Thus, substituting the wavelength dependence of $R(\lambda)$ and $\Delta \sigma(\omega) / \sigma(\omega)$ in a Hagen-Rubens relation we can accurately describe the MRE in metal-insulating multilayer and find the correlation between the magnetorefractive and magnetoresistive effects $(\omega \rightarrow 0)$. Combining (4) and (5), leads to a definition for a fractional change in magnetoreflectance as

$$
\frac{\Delta R}{R}=\frac{1-R}{R} \times \frac{\Delta \sigma(\omega)}{2 \sigma(\omega)}=-\frac{1-R}{R} \frac{P_{1}(\omega) P_{2}(\omega)}{1+P_{1}(\omega) P_{2}(\omega)} .
$$

This expression shows the proportionality between MRE and polarizations $P(\omega)$ in ferromagnetic/dielectric multilayer films. In the present analysis the reflectivity of the multilayer can be obtained using Fresnel's reflection and transmission coefficients [13]. The complex refractive index of CoFe thin film can be extracted from (2a). In the midinfrared region, where optical phonons can be excited, the dielectric function $\varepsilon_{d}$ of insulating $\mathrm{Al}_{2} \mathrm{O}_{3}$ can be approximated by a phenomenological Lorentz oscillator model [21, 22]:

$$
\varepsilon_{d}=\varepsilon_{\infty} \prod_{j} \frac{\nu_{\mathrm{LO} j}^{2}-v^{2}-i \nu \gamma_{\mathrm{LO} j}}{\nu_{\mathrm{TO} j}^{2}-\nu^{2}-i \nu \gamma_{\mathrm{TO} j}},
$$

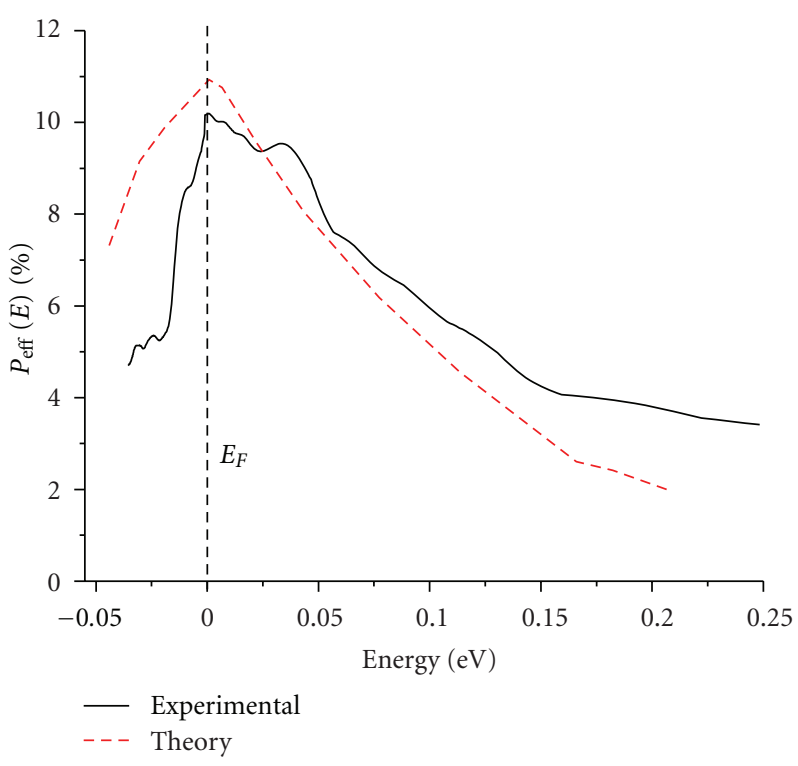

FIGURE 4: Effective tunnel spin polarization versus energy with respect to $E_{F}$, extracted from the MRE and reflectivity measurements for $\left[\mathrm{CoFe}(1.6 \mathrm{~nm}) / \mathrm{Al}_{2} \mathrm{O}_{3}(3 \mathrm{~nm})\right]_{10}$ multilayer films: experimental and simulated data.

where $\varepsilon_{\infty}$ is the high-frequency dielectric function. The vibrating frequencies and Lorentz widths of the LO and TO modes are $\nu_{\mathrm{LO} j}, \nu_{\mathrm{TO} j}$ and $\gamma_{\mathrm{LO} j}, \gamma_{\mathrm{TO} j}$, respectively. For our calculation only two pairs of phonon modes (LO/TO, $j=$ $1,2)$ were considered. The abovementioned parameters were taken from $[21,22]$.

Figure 3(b) displays the theoretical magnetoreflectance of the $\mathrm{CoFe} / \mathrm{Al}_{2} \mathrm{O}_{3}$ multilayer films as a function of the wavelength for different values of tunnel magnetoresistance (magnetic field), $\Delta \rho / \rho$ (Figure $2(\mathrm{a})$ ). The shape and spectral position of the dips for the MRE spectra (Figure 1(b)) are well described by our theoretical model (Figure 3(b)). In spectral region of $12-14 \mu \mathrm{m}$ the absolute value of $\Delta R / R$ becomes maximal.

It was shown above that the TMR dependence (Figure 2(a)) is connected only with the electron states near the Fermi level. For MRE there is a substantial contribution of electrons which tunnel from the occupied states below $E_{F}$ of one FM layer into the empty states above $E_{F}$ of the other FM layer. The effective polarization $P_{\text {eff }}(E)$ of the CoFe layer can be obtained by fitting the magnetoreflectance $\Delta R / R$ (Figure 3(b)) as a function of $\Delta \sigma(\omega) / \sigma(\omega)$ using (5). The experimental and simulated tunnelling spin-polarization spectra of the $\mathrm{CoFe} / \mathrm{Al}_{2} \mathrm{O}_{3}$ multilayers are shown in Figure 4. The experimental data for $P_{\text {eff }}(E)$ were obtained from optical reflectance, $R(\lambda)$ (inset of Figure 1(b)), and magnetoreflectance, $\Delta R / R$, dependences using the expression (6). It is worth noting that we determined the $P_{\text {eff }}(E)$ only in spectral region where the MRE has negative values. In this region we can use the Drude-like model for spin-dependent conductivities (1). Both experiment and theory suggest that the Fermi level falls in a region of maximal density of electron states. The measured and simulated spectra show 
a similar profile of $P_{\text {eff }}(E)$ around the Fermi level. However, some differences between simulated and measured data are observed. In particular, the simulated peak in $P_{\text {eff }}(E)$ at $E_{F}$ is sharper than in the experimental spectra. The measured $P_{\text {eff }}(E)$ seems displaced towards positive energy as compared to the simulated spectra (Figure 4). This discrepancy could be related to the features in $\Delta R / R$ and $R$ spectra which have been attributed to scattering of light at defects in amorphous $\mathrm{Al}_{2} \mathrm{O}_{3}$ matrix. The maximal values of $P_{\text {eff }}(E)$ extracted from MRE and TMR data (7), (3) at the Fermi level are in good agreement. The $P_{\text {eff }}(E)$ at $E_{F}$ is the largest $\left(P_{\text {eff }}\left(E_{F}\right) \approx 11 \%\right.$ from fitting TMR data) and is reduced below and above $E_{F}$. The value of $P_{\text {eff }}\left(E_{F}\right)$ exhibits small spin polarizations in comparison to pure CoFe alloy due to the particle oxidation of the CoFe surfaces. The $P_{\text {eff }}(E)$ is found to be most strongly reduced bellow $E_{F}$. The $P_{\text {eff }}(E)$ is associated with the filled (unfilled) states near (above) $E_{F}$ of the $\mathrm{CoFe} / \mathrm{Al}_{2} \mathrm{O}_{3}$ emitter or receiver interfaces. We determined the $P_{\text {eff }}(E)$ only for energies about $0.2 \mathrm{eV}$ below $E_{F}$ because of the negligible contribution to the tunnelling from the deepest electron states. Note that the spin-dependent tunnelling DOS of $\mathrm{Co} / \mathrm{Al}_{2} \mathrm{O}_{3}$ film extracted from the magnetocurrent of transistor-like devices and reported in $[23,24]$ shows a profile similar to our spectra (Figure 4).

\section{Conclusions}

We report experimental and theoretical study of the fielddependent IR magnetoreflectivity for $\mathrm{CoFe} / \mathrm{Cu}$ and $\mathrm{CoFe} /$ $\mathrm{Al}_{2} \mathrm{O}_{3}$ multilayer films. It was found a good correlation between GMR (TMR) and MRE as a function of the applied magnetic field which clearly displays the possibility of performing noncontact magnetotransport measurements by measuring the variation of the IR reflected from samples intensity of light at fixed wavelengths with high surface resolution (in case of focusing light to size of $\lambda$ ). The shape of the MRE curves as a function of wavelength is sensitive to the spin-dependent scattering parameters (in case of the GMR) and spin-dependent density of states at the $E_{F}$ for layered TMR films. Experimental results show that the MRE is sensitive to this fundamental parameter rather than just the magnitude of the magnetoresistance. The accurate fitting of the shape of the MRE curve as a function of wavelength allows determining the spin-dependent relaxation time, $\tau^{\dagger(\downarrow)}$, for films possessing GMR effect. The effective tunnel spin polarization $P_{\text {eff }}(E)$ versus energy can be extracted from the experimental dependences of MRE spectrum and reflectivity for $\mathrm{CoFe} / \mathrm{Al}_{2} \mathrm{O}_{3}$ multilayer films.

This study is of primary importance for applications of such materials in spintronics devices and magnetophotonic crystals.

\section{References}

[1] M. N. Baibich, J. M. Broto, A. Fert et al., "Giant magnetoresistance of (001)Fe/(001)Cr magnetic superlattices," Physical Review Letters, vol. 61, no. 21, pp. 2472-2475, 1988.

[2] G. Binasch, P. Grünberg, F. Saurenbach, and W. Zinn, "Enhanced magnetoresistance in layered magnetic structures with antiferromagnetic interlayer exchange," Physical Review B, vol. 39, no. 7, pp. 4828-4830, 1989.

[3] B. Dieny, B. A. Gurney, S. E. Lambert et al., "Magnetoresistive sensor based on the spin valve effect," US patent 5206590, 1993.

[4] C. Chappert, A. Fert, and F. N. van Dau, "The emergence of spin electronics in data storage," Nature Materials, vol. 6, no. 11, pp. 813-823, 2007.

[5] A. Fert, A. Barthelemy, and F. Petroff, "Spin transport in magnetic multilayers and tunnel junctions," in Nanomagnetism: Ultrathin Films, Multilayers and Nanostructures, D. M. Mills and J. A. C. Bland, Eds., chapter 6, Elsevier, Amsterdam, The Netherlands, 2006.

[6] J. C. Jacquet and T. Valet, "A new magnetooptical effect discovered on magnetic multilayers: the magnetorefractive effect," in Magnetic Ultrathin Films, Multilayers and Surfaces, E. Marinero, Ed., pp. 477-490, Materials Research Society, Pittsburg, Pa, USA, 1995.

[7] V. G. Kravets, D. Bozec, J. A. D. Matthew et al., "Correlation between the magnetorefractive effect, giant magnetoresistance, and optical properties of Co-Ag granular magnetic films," Physical Review B, vol. 65, no. 5, Article ID 054415, 9 pages, 2002.

[8] J. van Driel, F. R. De Boer, R. Coehoorn, and G. H. Rietjens, "Magnetic linear dichroism of infrared light in ferromagnetic alloy films," Physical Review B, vol. 60, no. 10, pp. R6949R6952, 1999.

[9] R. T. Mennicke, D. Bozec, V. G. Kravets, M. Vopsaroiu, J. A. D. Matthew, and S. M. Thompson, "Modelling the magnetorefractive effect in giant magnetoresistive granular and layered materials," Journal of Magnetism and Magnetic Materials, vol. 303, no. 1, pp. 92-110, 2006.

[10] A. B. Granovsky, I. V. Bykov, E. A. Gan'shina et al., "Magnetorefractive effect in magnetic nanocomposites," Journal of Experimental and Theoretical Physics, vol. 96, no. 6, pp. 11041112, 2003.

[11] V. G. Kravets, L. V. Poperenko, and A. F. Kravets, "Magnetoreflectance of ferromagnetic metal-insulator granular films with tunneling magnetoresistance," Physical Review B, vol. 79, no. 14, Article ID 144409, 2009.

[12] M. Vopsaroiu, D. Bozec, J. A. D. Matthew, S. M. Thompson, C. H. Marrows, and M. Perez, "Contactless magnetoresistance studies of $\mathrm{Co} / \mathrm{Cu}$ multilayers using the infrared magnetorefractive effect," Physical Review B, vol. 70, no. 21, Article ID 214423, 7 pages, 2004.

[13] M. Born and E. Wolf, Principles of Optics, Cambridge University Press, Cambridge, UK, 1999.

[14] A. V. Sokolov, Optical Properties of Metals, Blackie, Glasgow, UK, 1967.

[15] C. Kittel, Introduction to Solid State Physics, Wiley, New York, NY, USA, 1995.

[16] M. Julliere, "Tunneling between ferromagnetic films," Physics Letters A, vol. 54, no. 3, pp. 225-226, 1975.

[17] K. H. Bennemann, Ed., Nonlinear Optics in Metals, Clarendon Press, Oxford, UK, 1998.

[18] V. G. Kravets, D. Bozec, J. A. D. Matthew, and S. M. Thompson, "Calculation of the magnetorefractive effect in giant magnetoresistive granular films," Journal of Applied Physics, vol. 91, no. 10, pp. 8587-8590, 2002.

[19] J. C. Slonczewski, "Conductance and exchange coupling of two ferromagnets separated by a tunneling barrier," Physical Review B, vol. 39, no. 10, pp. 6995-7002, 1989.

[20] E. Y. Tsymbal, O. N. Mryasov, and P. R. LeClain, "Spindependent tunneling in magnetic tunnel junctions," Journal of Physics: Condensed Matter, vol. 15, pp. R109-R142, 2003. 
[21] M. Schubert, T. E. Tiwald, and C. M. Herzinger, "Infrared dielectric anisotropy and phonon modes of sapphire," Physical Review B, vol. 61, no. 12, pp. 8187-8201, 2000.

[22] V. G. Kravets, "Polaron interpretation of the magnetoreflectance effect in insulating $\alpha-\mathrm{Al}_{2} \mathrm{O}_{3}$," Physical Review B, vol. 72, no. 6, Article ID 064303, 2005.

[23] B. G. Park, T. Banerjee, J. C. Lodder, and R. Jansen, "Tunnel Spin polarization versus energy for clean and doped $\mathrm{Al}_{2} \mathrm{O}_{3}$ barriers," Physical Review Letters, vol. 99, no. 21, Article ID 217206, 2007.

[24] S. O. Valenzuela, D. J. Monsma, C. M. Marcus, V. Narayanamurti, and M. Tinkham, "Spin polarized tunneling at finite bias," Physical Review Letters, vol. 94, no. 19, Article ID 196601, 2005. 

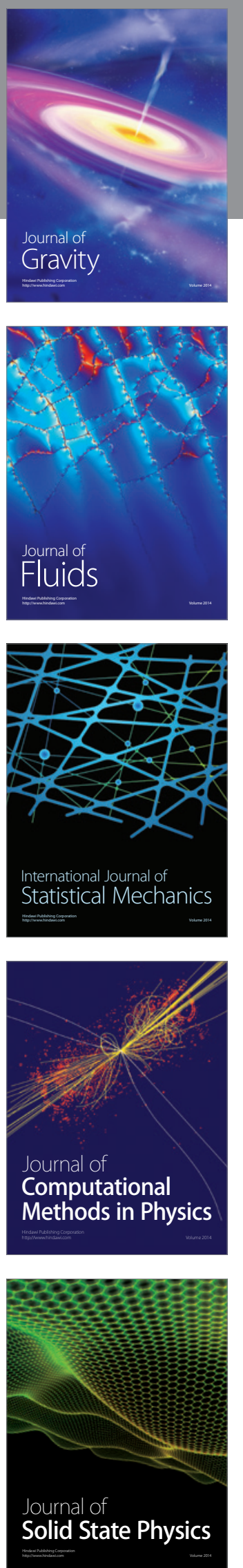

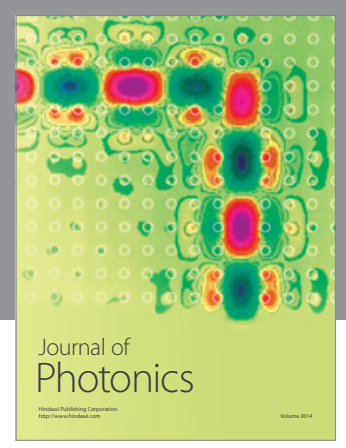

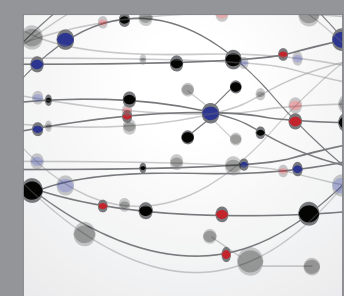

The Scientific World Journal
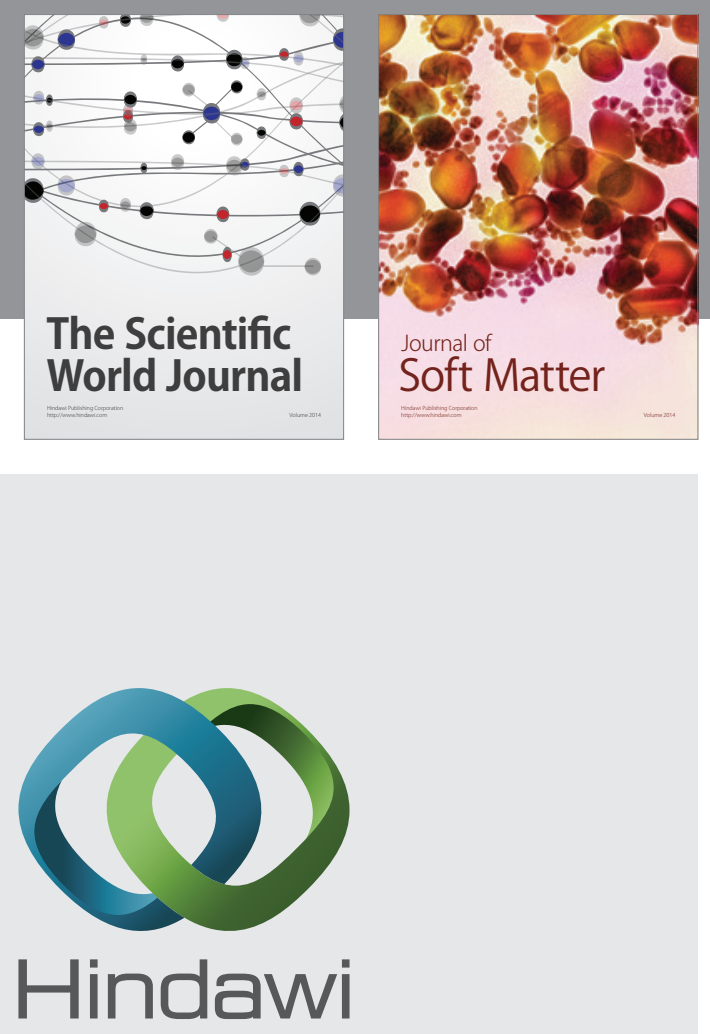

Submit your manuscripts at

http://www.hindawi.com
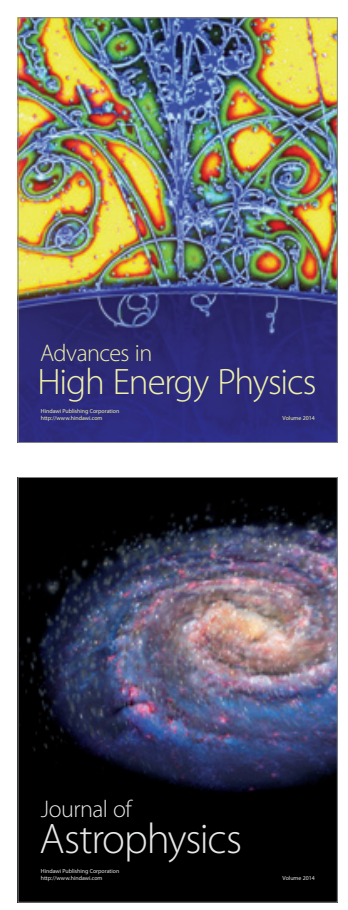
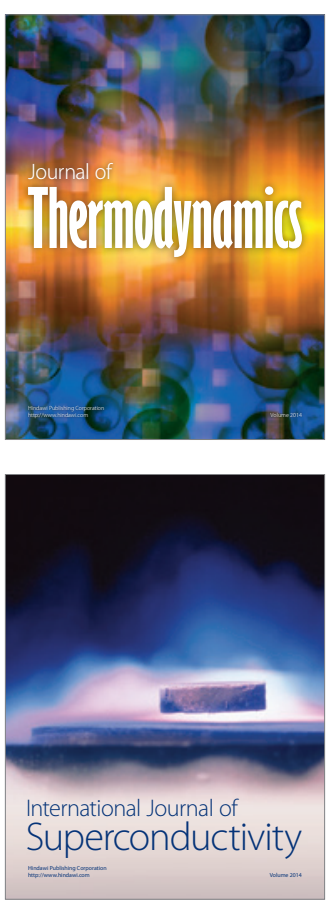
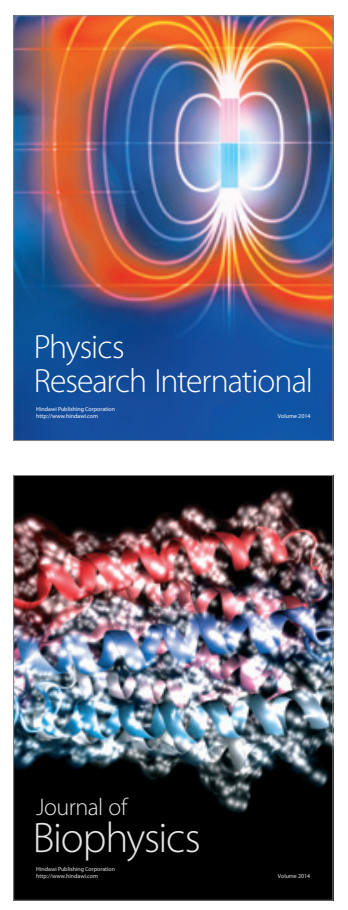
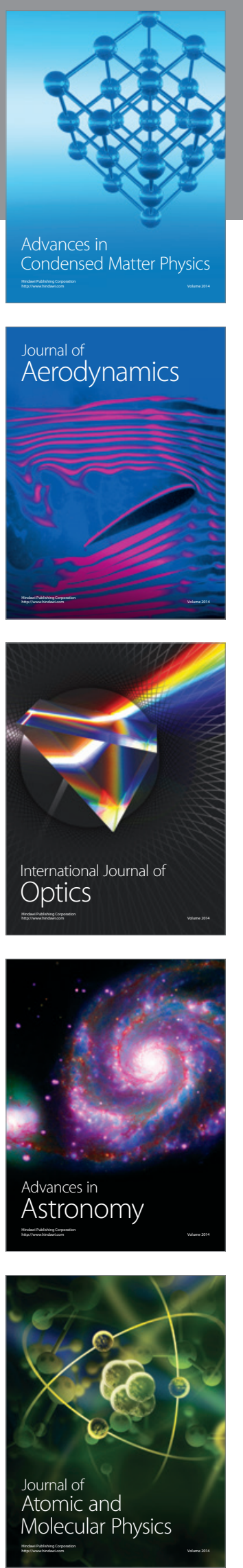